Supporting Information for

\title{
High Reversible Strain in Nanotwinned Metals
}

Suyun He, ${ }^{1}$ Binbin Jiang, ${ }^{1 *}$ Chunyang Wang, ${ }^{1}$ Chunjin Chen, ${ }^{1,2}$ Huichao Duan, $, 1,2$ Shuai Jin, ${ }^{1}$ Hengqiang Ye, ${ }^{1,3}$ Lei Lu, ${ }^{1} \mathrm{Kui} \mathrm{Du}^{1}$

${ }^{1}$ Shenyang National Laboratory for Materials Science, Institute of Metal Research, Chinese Academy of Sciences, Shenyang 110016, People's Republic of China

${ }^{2}$ School of Materials Science and Engineering, University of Science and Technology of China, Hefei, 230026, People's Republic of China

${ }^{3}$ Jihua Laboratory, Foshan,528251, China

*Corresponding author. Tel.: +86 248397 8628; fax: +86 2423891320.

E-mail address: bbjiang12s@imr.ac.cn 


\section{Contents}

Note 1. Loading rates of $\mathrm{Cu}$ films and Au nanorods

Note 2. The stress field of TEM samples under the fatigue tensile loading

Note 3. Bent twin lamellae in the nanotwinned $\mathrm{Cu}$ with the loading angle of $0^{\circ}$ after deformation

Note 4. The positive correlation between the apparent bending rate $(\psi)$ and the shear strain $\left(\varepsilon_{\mathrm{xy}}\right)$

Note 5. Experimental investigations on the matrix and thin twins in nanotwinned Cu before and after deformation

Note 6. Electron energy-loss spectroscopy (EELS) analysis on the TEM sample thickness of nanotwinned $\mathrm{Cu}$

Note 7. The types of dislocations in nanotwinned $\mathrm{Cu}$ and possible traces left by the dislocations

Note 8. Hard mode I dislocations nucleate from GBs and then impinge on TBs

Note 9. Discussion on the lattice rotation of the matrix and thin twin lamellae in nanotwinned $\mathrm{Cu}$

Note 10. Dislocations blocked by TBs in the Au nanorode 


\section{Note 1. Loading rates of $\mathrm{Cu}$ films and Au nanorods}

In the cyclic tension-tension loading tests, the loading rate of the nanotwinned $\mathrm{Cu}$ films ( $\left.\dot{\varepsilon}_{\mathrm{Cu}}\right)$ is roughly estimated as $1.2 \times 10^{-1} \mathrm{~s}^{-1}$ according to the formulas $\dot{\varepsilon}_{\mathrm{Cu}}=4 \mathrm{Af} / E$, where $A$ is the stress amplitude $(112.5 \mathrm{MPa}) f$ is the frequency $(30 \mathrm{~Hz}), E$ is the Young's modulus of the Ti alloy substrates (estimate as $112.5 \mathrm{GPa}$ ). In the in situ bending tests, the loading rate of the nanorods $\left(\dot{\varepsilon}_{\mathrm{Au}}\right)$ can be roughly estimated as $3 \times 10^{-3}-2 \times 10^{-2} \mathrm{~s}^{-1}$ according to the formula $\dot{\varepsilon}_{\mathrm{Au}}=\varepsilon_{\mathrm{m}} / t$. Here, $\varepsilon_{\mathrm{m}}$ is the maximum apparent bending rate of $\mathrm{Au}$ nanorods (ranging from 0.15 to 0.625 ) roughly corresponding to the shear strain, and $t$ is the time corresponding to the deformation, which ranges from 38 to 55 seconds. The loading rates of $\mathrm{Au}$ nanorods and $\mathrm{Cu}$ films show that they are both deformed under nearly quasi-static deformation conditions. Therefore, the difference of the loading rates between $\mathrm{Au}$ nanorods and $\mathrm{Cu}$ films was not too significant to induce a dramatic variation of deformation mechanism.

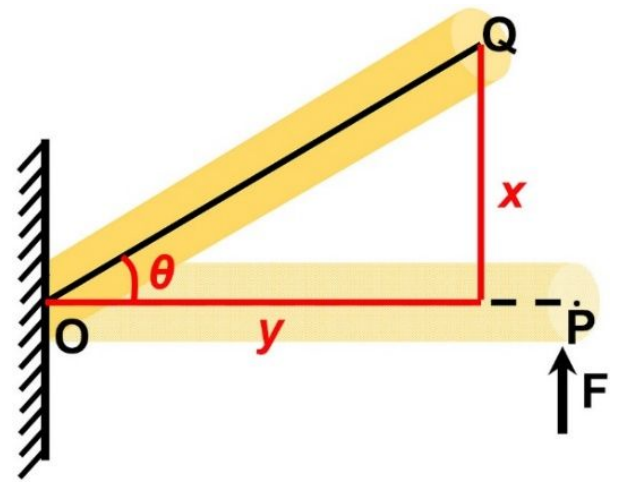

Figure S1. Schematic illustration of the bending deformation of a nanorod. $O$ is the fixed end of the nanorod, $\mathrm{Q}$ is the ending position of the free end of the nanorod, $x$ is the normal displacement of the free end of nanorods from the original long axis of nanorods, $y$ is the length of the nanorods in projection on the original long axis. $\mathrm{F}$ indicates the applied force on the nanorod during the bending test. 


\section{Note 2. The stress field of TEM samples under the fatigue tensile loading}

In order to avoid possible damage on the observation areas during the fatigue tests, the TEM samples were glued onto thin rings, and then mounted on substrates through these rings ${ }^{1-2}$. During the fatigue tensile deformation, the strain of the substrates is transferred to the samples through the thin rings, resulting in the fatigue deformation of the samples. Notably, perforations are introduced in the center of the samples by ionmilling as the standard procedure for preparing TEM samples. The normal and shear stresses both rise gradually with the increasing distance from the perforation, reach the maximum and then fall off beyond the point, according to the finite element method calculations on the freestanding metal thin film with $170 \mathrm{~nm}$ thickness ${ }^{3}$. These stresses in the sample are likely the reason for the bending of the twin lamellae observed in this work. Some irregular shape of perforations introduced by ion milling may causes some inhomogeneous stress distribution in the samples.

Note 3. Bent twin lamellae in the nanotwinned $\mathrm{Cu}$ with the loading angle of $0^{\circ}$ after deformation

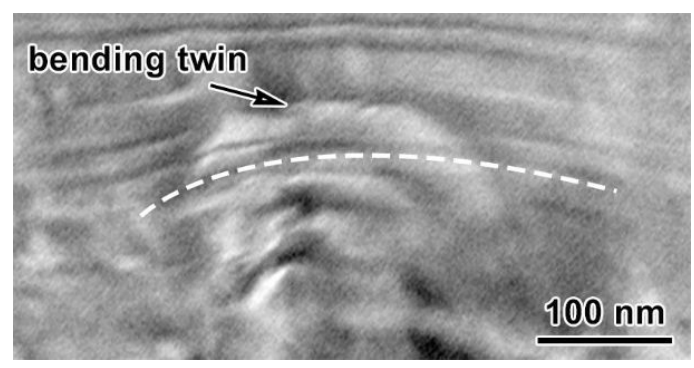

Figure S2. HAADF-STEM image of the nanotwinned $\mathrm{Cu}$ with the loading angle of $0^{\circ}$ after deformation. The black arrow indicates a bent twin lamella, while a bent TB is indicated by the white dashed line. 
Note 4. The positive correlation between the apparent bending rate $(\psi)$ and the shear strain $\left(\varepsilon_{\mathrm{xy}}\right)$

Considered bent twin lamellae in nanotwinned $\mathrm{Cu}$ as bent objects with both atomic-scale and microscale images, the apparent bending rate $\psi$ and the shear strain $\varepsilon_{\mathrm{xy}}$ of the dislocation-free bent twin lamellae were both measured from experimental images and shown in Fig. S3. The statistical distribution of $\psi$ and $\varepsilon_{\mathrm{xy}}$ against twin thickness shows that the upper limits of $\psi$ and $\varepsilon_{\mathrm{xy}}$ follow similar trend that they increase with the decrease of twin thickness. Thus, $\psi$ is positively correlated with $\varepsilon_{\mathrm{xy}}$, although its value is not directly equivalent to the shear strain.

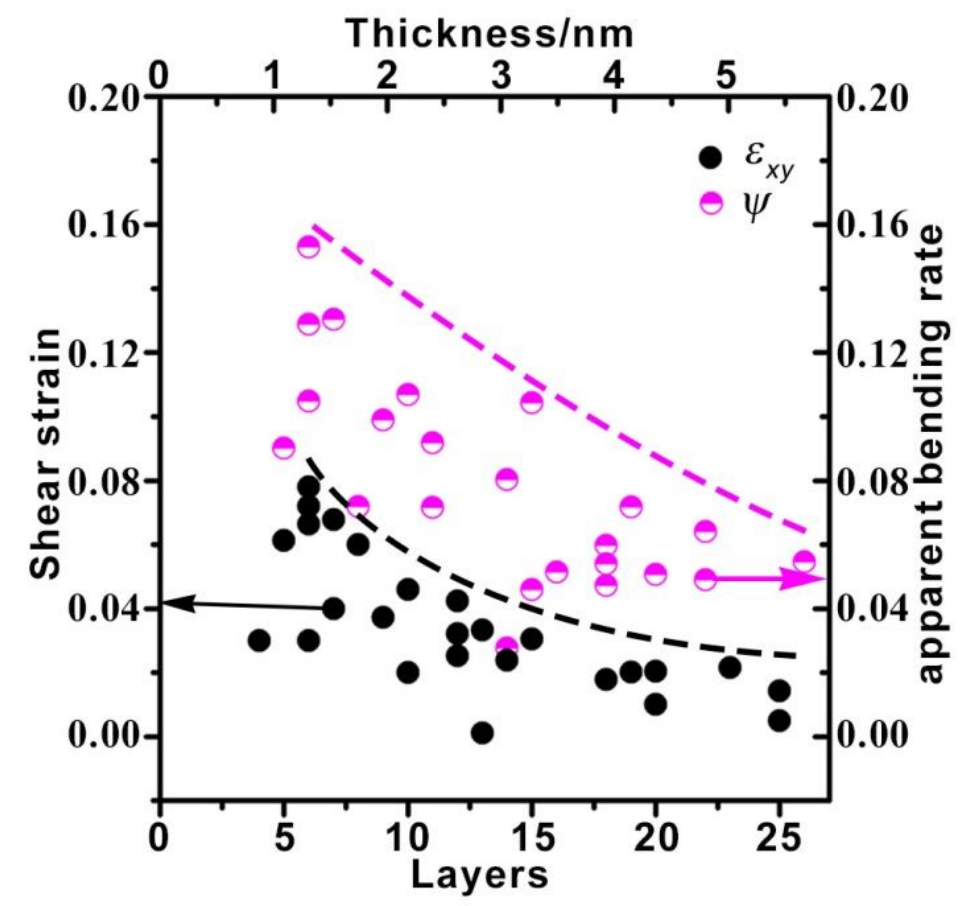

Figure S3. Statistical distribution of measured shear strains in dislocation-free twin lamellae and the apparent bending rate of these twin lamellae with different thickness in nanotwinned $\mathrm{Cu}$. The black and magenta dashed lines are the upper boundaries of the shear strains and apparent bending rate, respectively. 


\section{Note 5. Experimental investigations on the matrix and thin twins in nanotwinned}

\section{$\mathrm{Cu}$ before and after deformation}

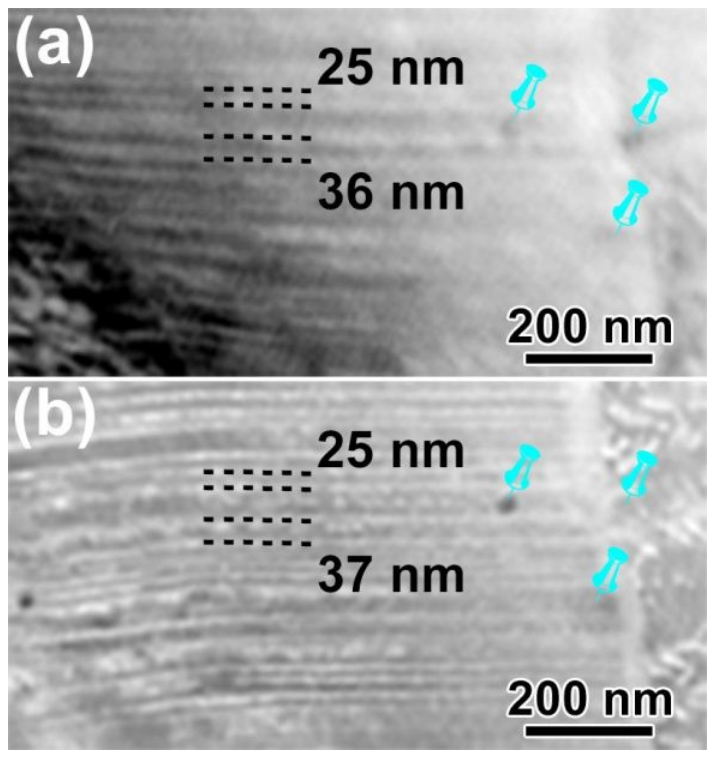

Figure S4. HAADF-STEM images of nanotwinned $\mathrm{Cu}$ before (a) and after (b) fatigue tensile deformation in the same region. The cyan markers in (a) and (b) are used to determine the corresponding positions in the sample.

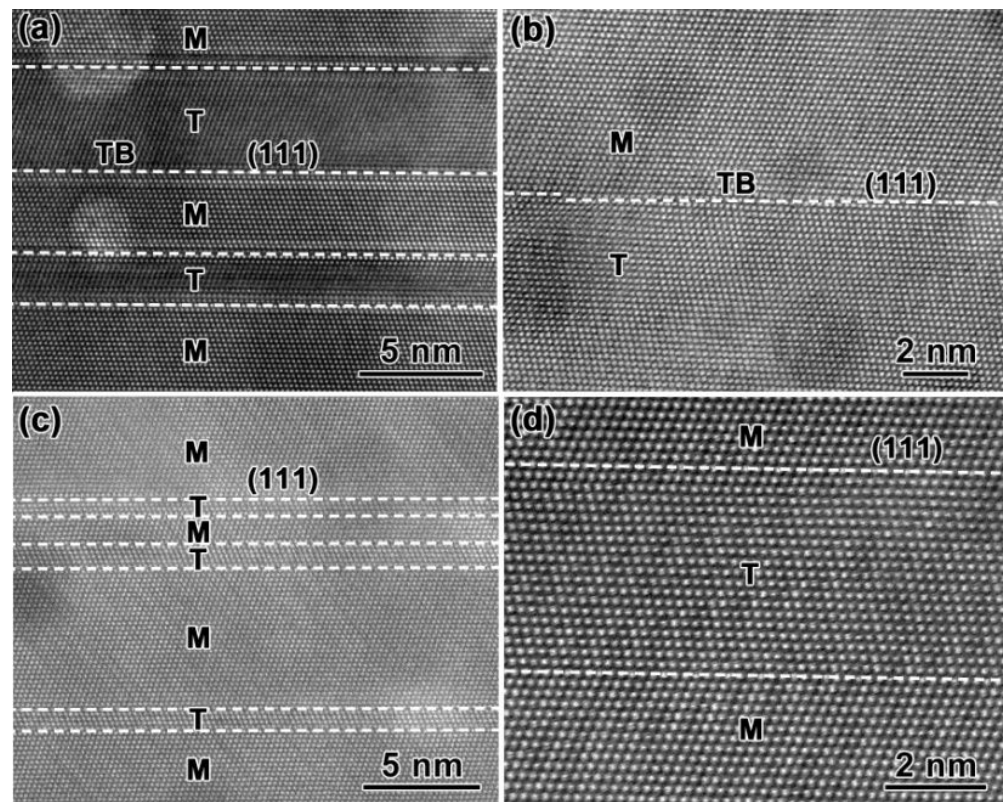

Figure S5. (a) Atomic resolution HRTEM image taken along the [11̄0] axis of nanotwinned $\mathrm{Cu}$ before deformation. (b-d) Atomic resolution HAADF-STEM images taken along the [110] axis of nanotwinned $\mathrm{Cu}$ before deformation. TBs are indicated by white dashed lines. 
To further verify the absence of lattice dislocations inside thin twin lamellae with thickness less than $5 \mathrm{~nm}$, two-beam bright-field imaging experiments were carried out in TEM based on the invisibility criterion $\boldsymbol{g} . \boldsymbol{b}=0$, as shown in Fig. S6 and Table S1. Note that Table S1 shows only the analysis for full dislocations in the twin lamellae, considering that Shockley partial dislocations will be easily observed through atomic resolution HRTEM or HAADF-STEM images. As seen in Table S1, all full dislocations can be visible in two-beam bright-field images with one or more diffraction vectors ( $g$ ). Therefore, considering that no dislocations were observed in thin twin lamellae with any $\boldsymbol{g}$ (Fig. S6b-d), we consider that no dislocations were activated inside these twin lamellae during deformation. In contrast, dislocations were often observed in thick twin lamellae, as indicated by arrows in Figs. S6b-d. Notably, in TEM images with the zone axis close to [110] (Figs. S6b-c), bowed (or curved) dislocations indicated by green arrows are probably hard mode II dislocations, while straight dislocations indicated by red arrows are hard mode I dislocations.

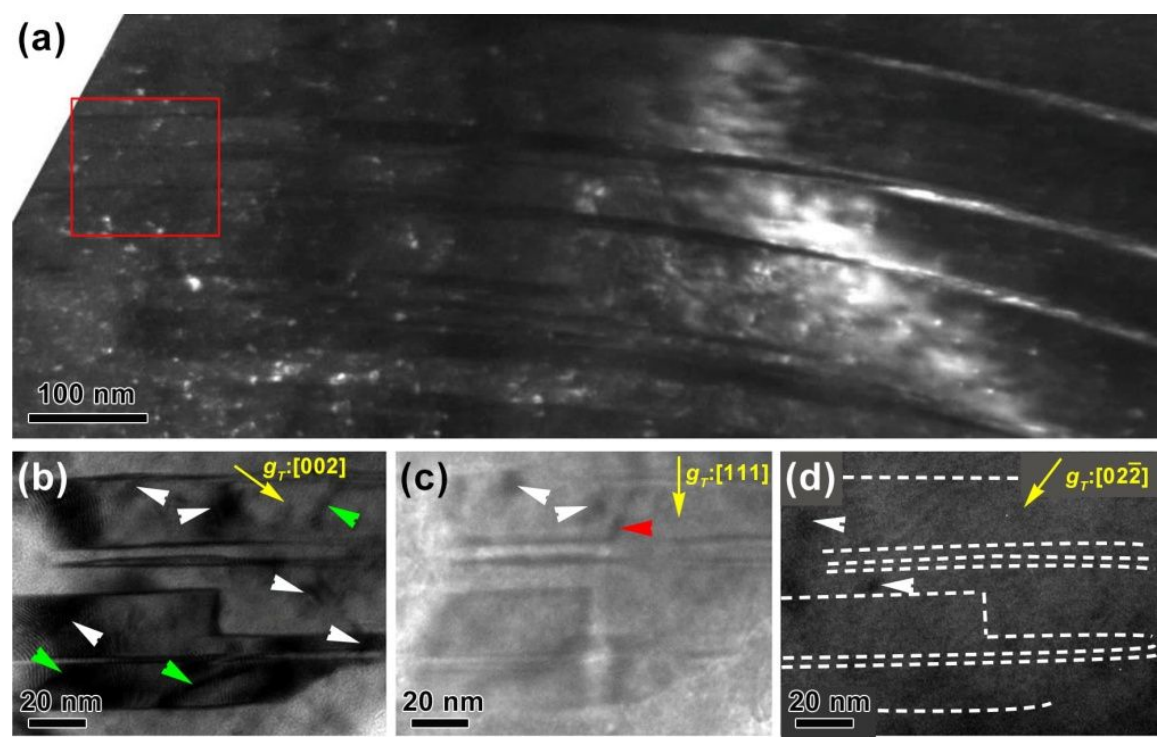

Figure S6. (a) Dark-field TEM image of nanotwinned $\mathrm{Cu}$ after deformation. (b-d) Two-beam bright-field images of the framed area in (a) under (b) $\boldsymbol{g}_{T}=002$, (c) $\boldsymbol{g}_{T}=111$ and $\boldsymbol{g}_{T}=02 \overline{2}$. The yellow arrows indicate the direction of $\boldsymbol{g}_{T}$. Dislocations are indicated by white arrows, while typical 
hard mode I and hard mode II dislocations are indicated by red and green arrows, respectively. White dashed lines in (d) denote TBs.

Table S1. The visibility (v) and invisibility (i) of full dislocations with Burgers vectors of $1 / 2<110>$ under different two beam conditions.

\begin{tabular}{|c|c|c|c|c|c|}
\hline Type & Slip plane & $\begin{array}{c}\text { Burgers } \\
\text { vector }\end{array}$ & $\mathbf{0 0 2}_{\mathrm{T}}$ & $111_{\mathrm{T}}$ & $02 \overline{2}_{\mathrm{T}}$ \\
\hline \multirow{9}{*}{ Hard mode } & \multirow{3}{*}{$(11 \overline{1})$} & $1 / 2[101]$ & $\mathrm{v}$ & $\mathrm{v}$ & $\mathrm{v}$ \\
\hline & & $1 / 2[011]$ & $\mathrm{v}$ & $\mathrm{v}$ & $\mathrm{i}$ \\
\hline & & $1 / 2[\overline{1} 10]$ & $\mathrm{i}$ & $\mathrm{i}$ & $\mathrm{v}$ \\
\hline & \multirow{3}{*}{ (1̄11) } & $1 / 2[110]$ & $\mathrm{i}$ & $\mathrm{v}$ & $\mathrm{v}$ \\
\hline & & $1 / 2[101]$ & $\mathrm{v}$ & $\mathrm{v}$ & $\mathrm{v}$ \\
\hline & & $1 / 2[0 \overline{1} 1]$ & $\mathrm{v}$ & $\mathrm{i}$ & $\mathrm{v}$ \\
\hline & \multirow{3}{*}{$(1 \overline{1} 1)$} & $1 / 2[011]$ & $\mathrm{v}$ & $\mathrm{v}$ & $\mathrm{i}$ \\
\hline & & $1 / 2[\overline{1} 01]$ & $\mathrm{v}$ & $\mathrm{i}$ & $\mathrm{v}$ \\
\hline & & $1 / 2[110]$ & $\mathrm{i}$ & $\mathrm{v}$ & $\mathrm{v}$ \\
\hline \multirow{3}{*}{ Soft mode } & \multirow{3}{*}{ (111) } & $1 / 2[\overline{1} 10]$ & $\mathrm{i}$ & $\mathrm{i}$ & $\mathrm{v}$ \\
\hline & & $1 / 2[01 \overline{1}]$ & $\mathrm{v}$ & $\mathrm{i}$ & $\mathrm{v}$ \\
\hline & & $1 / 2[\overline{1} 01]$ & $\mathrm{v}$ & $\mathrm{i}$ & $\mathrm{v}$ \\
\hline
\end{tabular}

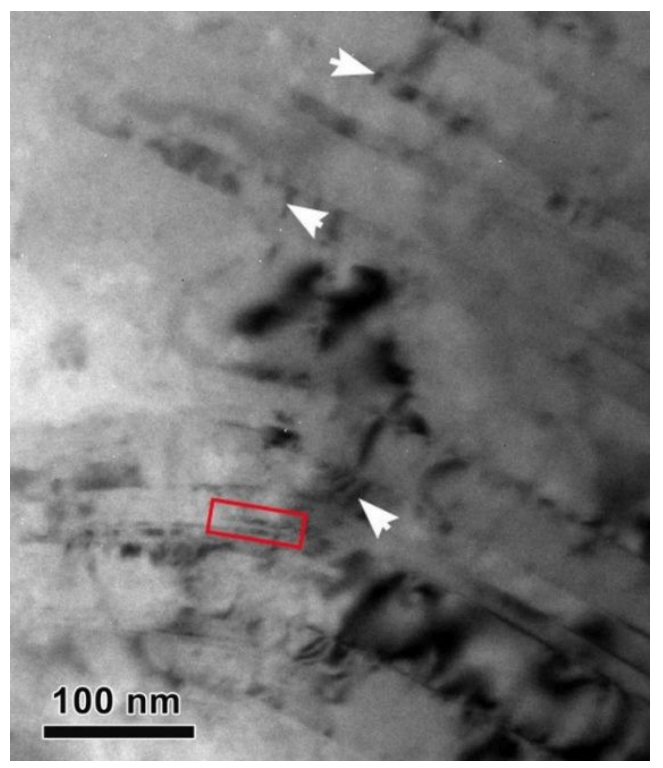

Figure S7. Bright-field TEM image of nanotwinned $\mathrm{Cu}$ after deformation. White arrows indicate dislocations inside thick twin lamellae. The red box indicates a dislocation-free thin twin lamella. 

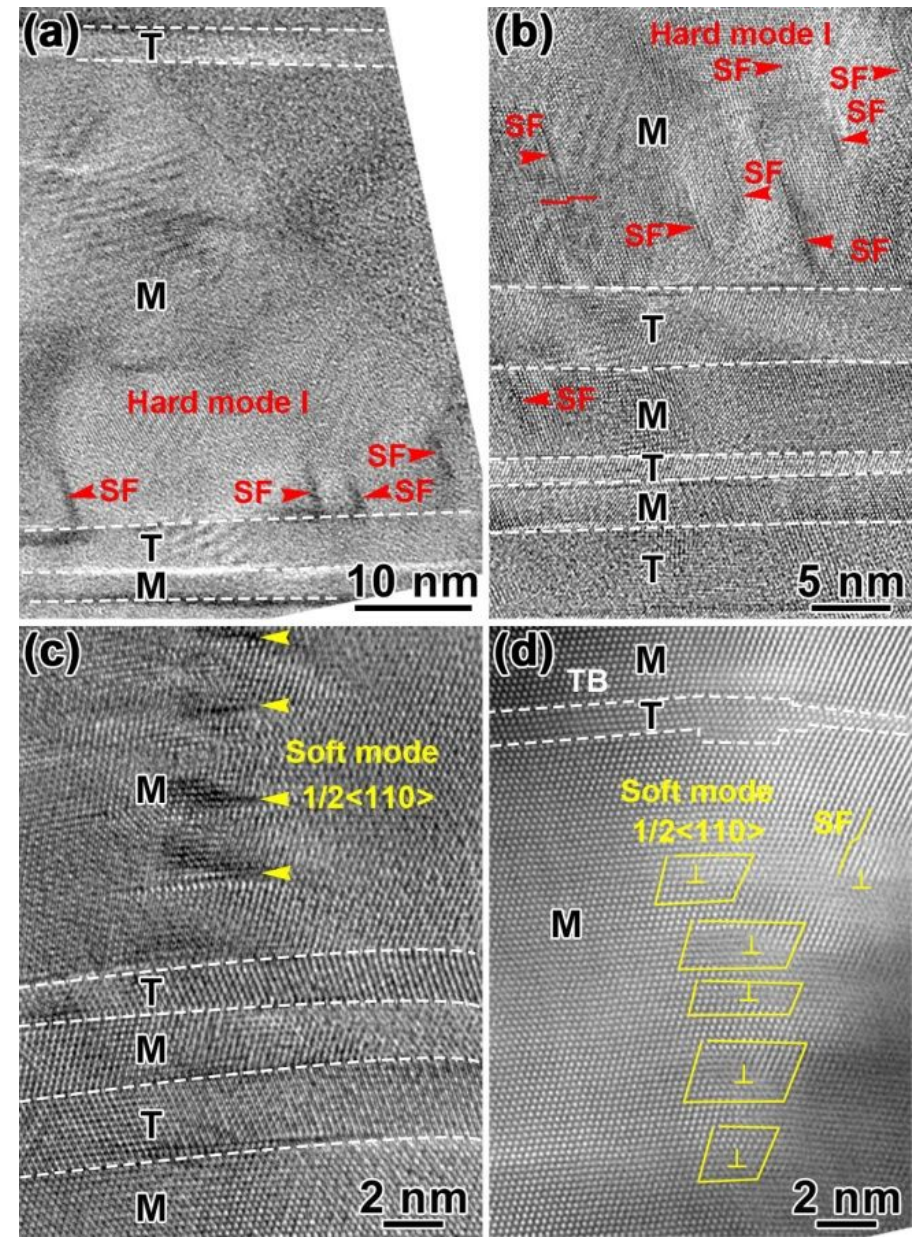

Figure S8. (a) Bright-field TEM image of the nanotwinned $\mathrm{Cu}$ after deformation. (b-c) Atomic resolution HRTEM images taken along the [110] axis of nanotwinned $\mathrm{Cu}$ after deformation. (d) Atomic resolution HAADF-STEM image taken along the [110] axis of the nanotwinned $\mathrm{Cu}$ after deformation. Hard mode I partial dislocations and SFs they induced are indicated by red arrows. Soft mode dislocations are indicated by yellow arrows. The Burgers vector of the dislocations that constitute the dislocation wall is $1 / 2<110>$. TBs are indicated by white dashed lines.

Note 6. Electron energy-loss spectroscopy (EELS) analysis on the TEM sample thickness of nanotwinned $\mathrm{Cu}$ 


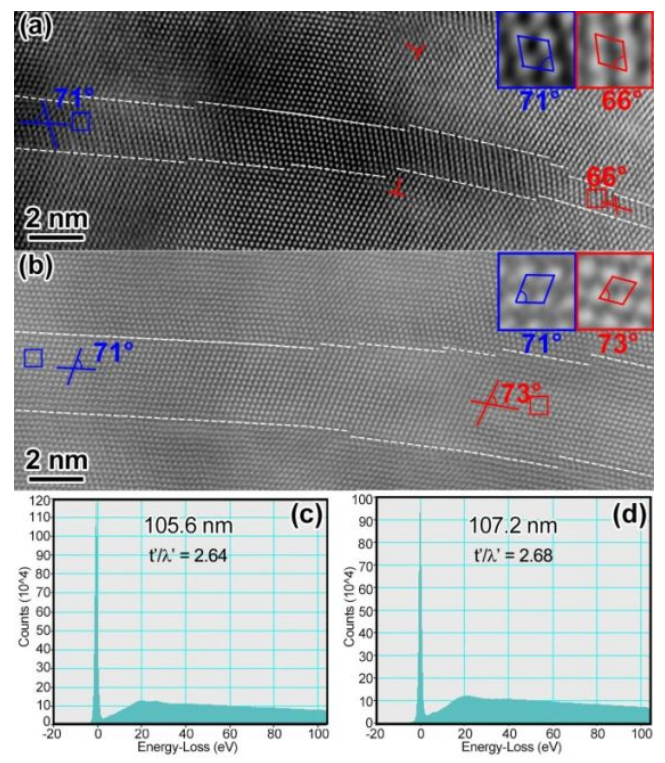

Figure S9. (a-b) Atomic resolution HAADF-STEM images taken along the [110] axis of the nanotwinned $\mathrm{Cu}$ after deformation. The insets are the enlarged atomic resolution images from the areas indicated by blue and red boxes in (a-b). TBs are indicated by white dashed lines. (c-d) Electron energy-loss spectra (EELS) show the sample thickness ( $\mathrm{t}^{\prime}$ ) is $105.6 \mathrm{~nm}$ in (a) and $107.2 \mathrm{~nm}$ in (b), respectively. Here, the mean free path $\left(\lambda^{\prime}\right)$ is $40 \mathrm{~nm}$.
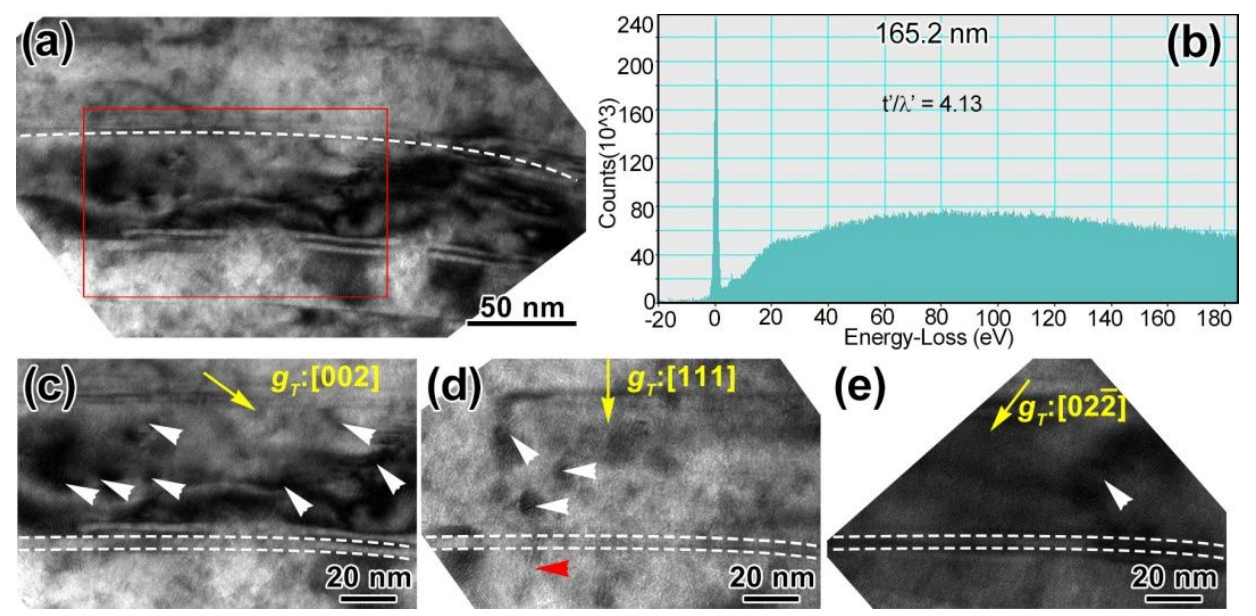

Figure S10. (a) Bright-field TEM image of nanotwinned $\mathrm{Cu}$ after deformation. (b) EELS shows the sample thickness is $165.2 \mathrm{~nm}$ in (a) with $\lambda^{\prime}=40 \mathrm{~nm}$. (c-e) Two-beam condition images of the framed area in (a) under (b) $\boldsymbol{g}_{T}=002$, (c) $\boldsymbol{g}_{T}=111$ and $\boldsymbol{g}_{T}=02 \overline{2}$. The yellow arrows indicate the direction of $\boldsymbol{g}_{T}$. White arrows indicate dislocations. The red arrow indicates a typical hard mode I dislocation. White dashed lines indicate TBs. 

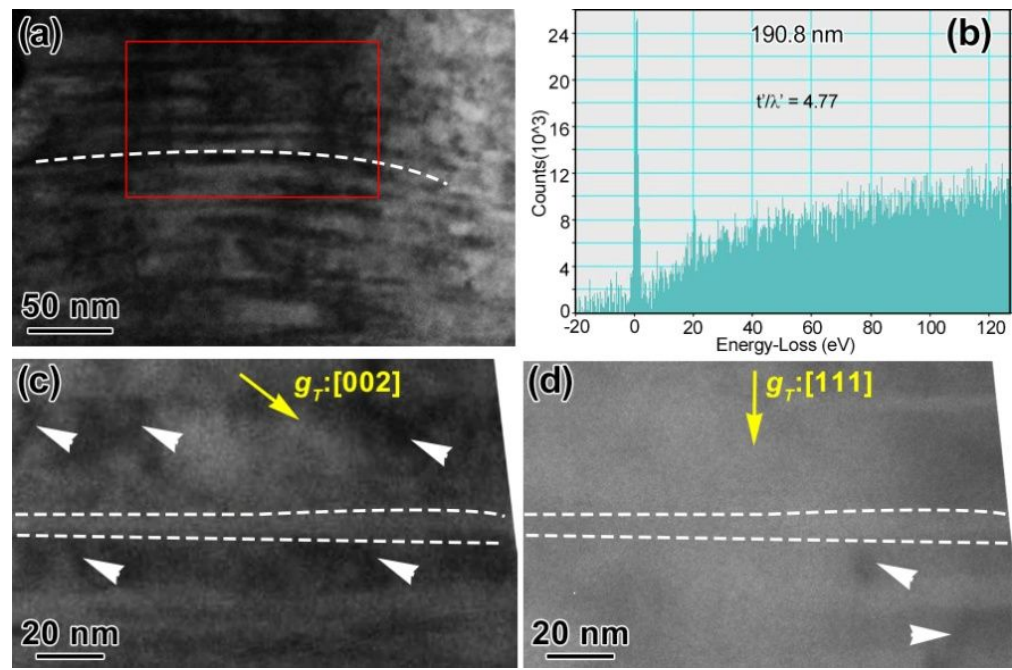

Figure S11. (a) Bright-field TEM image of nanotwinned $\mathrm{Cu}$ after deformation. (b) EELS shows the sample thickness is $190.8 \mathrm{~nm}$ in (a) with $\lambda^{\prime}=40 \mathrm{~nm}$. (c-d) Two-beam condition TEM images of the framed area in (a) under (c) $\boldsymbol{g}_{T}=002$ and (d) $\boldsymbol{g}_{T}=111$. The yellow arrows indicate the direction of $\boldsymbol{g}_{T}$. White arrows indicate dislocations. White dashed lines indicate TBs.
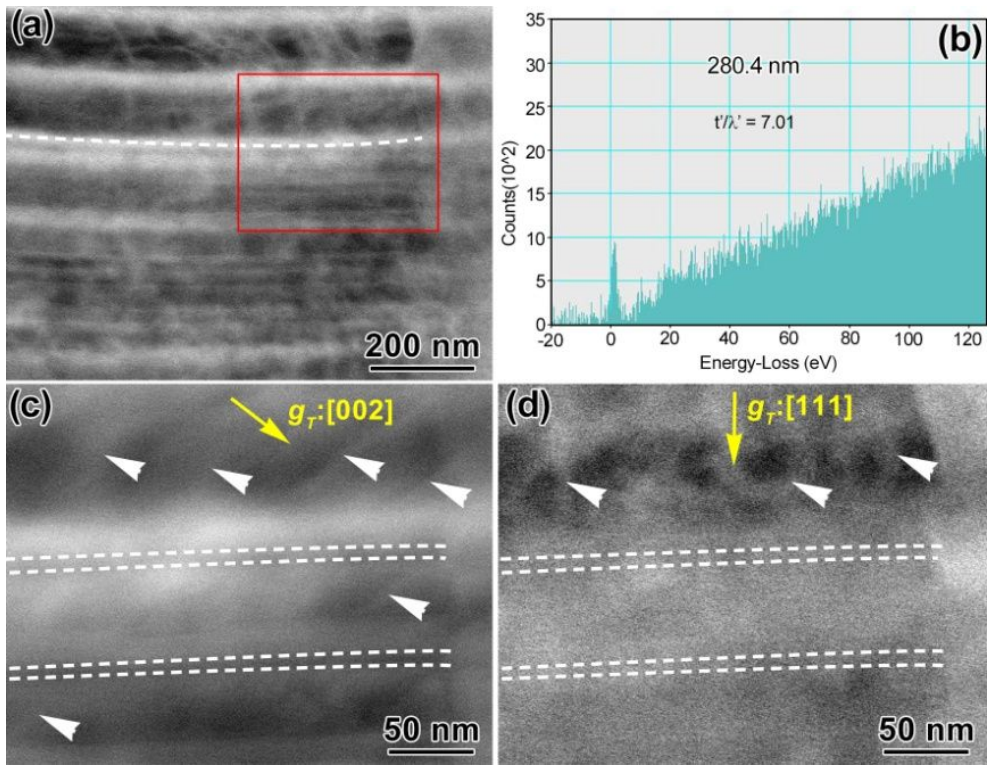

Figure S12. (a) HAADF-STEM image of nanotwinned $\mathrm{Cu}$ after deformation. (b) EELS show the sample thickness is $280.4 \mathrm{~nm}$ in (a) with $\lambda^{\prime}=40 \mathrm{~nm}$. (c-d) HAADF-STEM images of the framed area in (a) under two-beam condition of (c) $\boldsymbol{g}_{T}=200$ and (d) $\boldsymbol{g}_{T}=111$. The yellow arrows indicate the direction of $\boldsymbol{g}_{T}$. White arrows indicate dislocations. White dashed lines indicate TBs. 
Note 7. The types of dislocations in nanotwinned $\mathrm{Cu}$ and possible traces left by the

\section{dislocations}

Table S2. The types of dislocations in nanotwinned $\mathrm{Cu}$ and possible traces left by the dislocations.

(Dislocations on $\mathrm{ABD}, \mathrm{BCD}$, and $\mathrm{CAD}$ planes are equivalent, thus, among them, only the dislocations on $\mathrm{ABD}$ plane are listed in the table for simplicity.)

\begin{tabular}{|c|c|c|c|c|}
\hline Type & Slip plane & Slip direction & Burgers vector & Traces of dislocations left during gliding \\
\hline \multirow{3}{*}{$\begin{array}{l}\text { Hard mode } \\
\qquad \text { I }\end{array}$} & \multirow{3}{*}{$\begin{array}{l}\mathrm{ABD} \\
(11 \overline{1})\end{array}$} & \multirow{3}{*}{$\begin{array}{l}\text { inclined to the } \\
\text { CTB (ABC) }\end{array}$} & $\begin{array}{ll}\text { DA } & 1 / 2[101] \\
\text { DB } & 1 / 2[011]\end{array}$ & $\begin{array}{l}\text { 1. blocked at the CTB } \\
\text { 2. transits and leaves a sessile i-lock at the } \\
\text { CTB (Acta Mater., 2008, 56, 1126) } \\
\text { 3. transits and leaves a Shockley } \\
\text { dislocation as a step on the CTB (Acta } \\
\text { Mater., 2008, 56, 1126) }\end{array}$ \\
\hline & & & $\mathrm{AB} \quad 1 / 2[110]$ & $\begin{array}{l}\text { 1. blocked at the CTB } \\
\text { 2. transits and leaves no defects at the } \\
\text { CTB (Acta Mater., 2006, } 54,1163 \text { ) }\end{array}$ \\
\hline & & & $\begin{array}{ll}\mathrm{D} \gamma & 1 / 6[112] \\
\gamma \mathrm{A} & 1 / 6[2 \overline{1} 1] \\
\gamma \mathrm{B} & 1 / 6[\overline{1} 21]\end{array}$ & $\begin{array}{l}\text { transits and leaves a stacking fault inside } \\
\text { the twin lamella }\end{array}$ \\
\hline $\begin{array}{l}\text { Hard mode } \\
\quad \text { II }\end{array}$ & $\begin{array}{l}\mathrm{ABD} \\
(11 \overline{1})\end{array}$ & $\mathrm{AB}$ & $\begin{array}{ll}\mathrm{DA} & 1 / 2[101] \\
\mathrm{DB} & 1 / 2[011] \\
\mathrm{AB} & 1 / 2[\overline{1} 10]\end{array}$ & $\begin{array}{l}\text { 1. leaves a straight dislocation on the } \\
\text { CTB }^{4} \\
\text { 2. leaves no defects at the CTB }\end{array}$ \\
\hline \multirow{2}{*}{ Soft mode } & \multirow{2}{*}{$\begin{array}{l}\mathrm{ABC} \\
(111)\end{array}$} & \multirow{2}{*}{$\begin{array}{l}\text { parallel to the } \\
\text { CTB (ABC) }\end{array}$} & $\begin{array}{ll}\mathrm{AB} & 1 / 2[\overline{1} 10] \\
\mathrm{AC} & 1 / 2[01 \overline{1}] \\
\mathrm{CB} & 1 / 2[\overline{1} 01]\end{array}$ & no interaction with the CTB \\
\hline & & & $\begin{array}{lc}\mathrm{A} \delta & 1 / 6[\overline{1} 2 \overline{1}] \\
\delta \mathrm{B} & 1 / 6[\overline{2} 11] \\
\delta \mathrm{C} & 1 / 6[1 \overline{1}]\end{array}$ & $\begin{array}{l}\text { forms a step on the CTB as a Shockley } \\
\text { dislocation }\end{array}$ \\
\hline
\end{tabular}




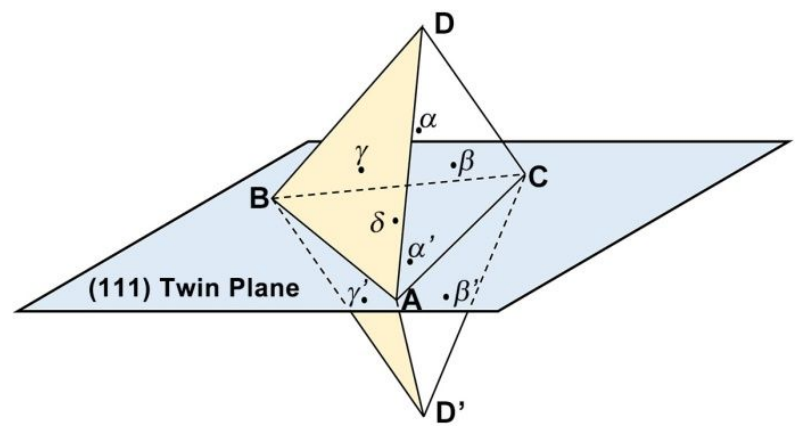

Figure S13. An illustration of double Thompson tetrahedron.

Note 8. Hard mode I dislocations nucleate from GBs and then impinge on TBs
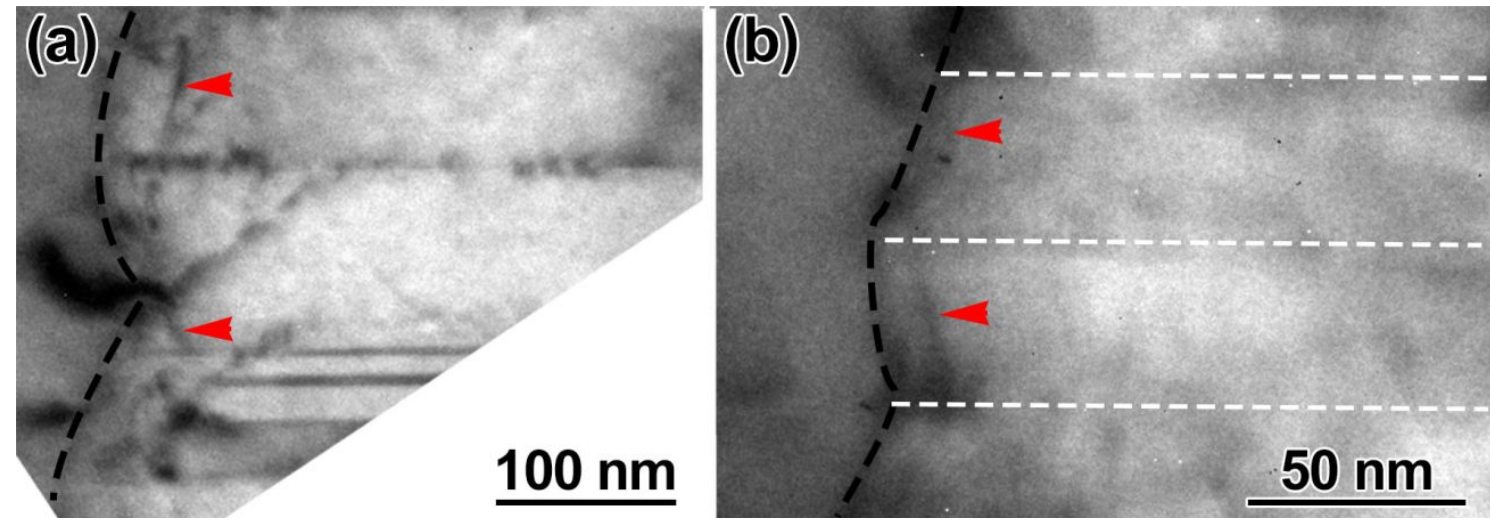

Figure S14. (a-b) Bright-field TEM images of nanotwinned $\mathrm{Cu}$ after deformation. Red arrows indicate hard mode I dislocations. GBs are indicated by black dashed lines. TBs in (b) are indicated by white dashed lines.

Note 9. Discussion on the lattice rotation of the matrix and thin twin lamellae in nanotwinned $\mathrm{Cu}$

The angles $(\omega)$ of $(11 \overline{1})$ lattice planes in both the matrix and the twin were measured in Fig. $2 b$ also as Fig. S15a. Here, $\omega$ is defined as the angle between the indicated (11) $)$ planes (Fig. S15a) and x-axis (horizontal direction). The lattice rotation angles of the matrix and twin across the image are described as $\Delta \omega_{\mathrm{M}}=\omega_{\mathrm{B}}-\omega_{\mathrm{A}}$ and $\Delta \omega_{\mathrm{T}}$ 
$=\omega_{D}-\omega_{C}$, respectively. The measured results show that both lattices of the matrix and the twin are rotated, but $\Delta \omega_{\mathrm{M}}$ is larger as approximately $11^{\circ}$ while $\Delta \omega_{\mathrm{T}}$ is smaller as approximately $6^{\circ}$. It should be noted that two dislocations with the Burgers vector of $1 / 2[110]$ are observed in the matrix. The rotation of the lattice plane induced by the dislocations can be roughly estimated according to the formula $\Delta \omega_{\text {dis }}=n b / T$. Here $n$ is the number of dislocations in the matrix ( $n=2$ here), $b$ is the Burgers vector of the dislocations, and $T$ is the thickness of the matrix $(\sim 5 \mathrm{~nm})$. Therefore, $\Delta \omega_{\mathrm{dis}}$ is approximately $6^{\circ}$, which implies that the lattice rotation of the matrix is partially caused by the dislocations inside.

The distribution of normal strain along [11 $]$ direction in (111) plane was also analyzed using the lattice distortion analysis (LADIA) program (Fig. S15b). The result shows that a tensile strain $\left(\varepsilon_{\mathrm{Mu}}\right)$ exists at the upper part of the matrix and a compressive strain $\left(\varepsilon_{\mathrm{Ml}}\right)$ at the lower part, which is consistent with the characteristic of a bending lamella. The rotation of the lattice plane induced by the normal strain can be roughly estimated as approximately $3.7^{\circ}$, according to the formula $\Delta \omega_{\text {nor }}=\left(\overline{\varepsilon_{\mathrm{Mu}}}-\overline{\varepsilon_{\mathrm{Ml}}}\right) L / T$. Here, $\overline{\varepsilon_{\mathrm{Mu}}}$ is the average tensile strain of the upper part of the matrix, $\overline{\varepsilon_{\mathrm{Ml}}}$ is the average compressive strain of the lower part of the matrix, $L$ is the length of the lamella $(\sim 12.5$ $\mathrm{nm})$ and $T$ is $\sim 5 \mathrm{~nm}$. Thus the normal strain can also lead to part of the lattice rotation of the matrix. The magnitude of normal strain in the matrix is less than $3 \%$. Such normal strain retained in the matrix may be caused by the constraint of surrounding grains. Meanwhile, the maximum normal strain measured in the twin lamella is less than $1 \%$, which is far less than its maximum shear strain ( $7.8 \%$, as shown in Fig. $2 \mathrm{f})$. The $\Delta \omega_{\text {nor }}$ 
in the twin lamella is roughly estimated as $4.5^{\circ}$, where $L$ is $\sim 12.5 \mathrm{~nm}$ and the average twin thickness is $\sim 1.5 \mathrm{~nm}$. Therefore, the rotation of lattice in the matrix was caused by the dislocations and retained normal strain inside the matrix, while the rotation of lattice in the twin lamella was mainly caused by the retained normal strain.
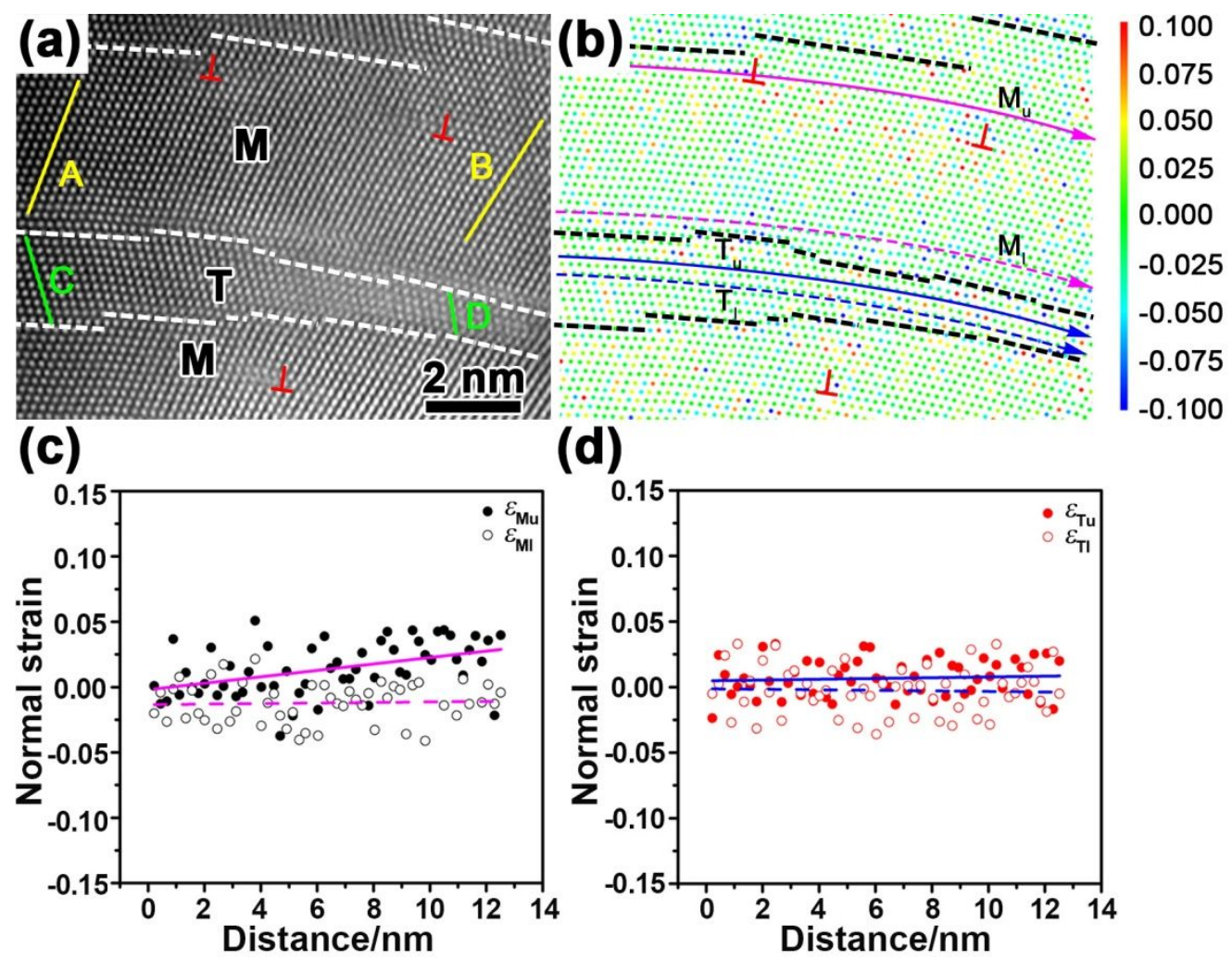

Figure S15. (a) Atomic resolution HAADF-STEM image of a bent twin lamella in nanotwinned $\mathrm{Cu}$ after deformation. (b) Lattice normal strain along $1 / 4[11 \overline{2}]_{\mathrm{T}}$ in $(111)_{\mathrm{T}}$ plane and $1 / 4[11 \overline{2}]_{\mathrm{M}}$ in (111) м plane, determined from (a), analyzed using the LADIA program. The positive values represent tensile strain and negative values represent compressive strain. (c-d) The distribution of normal strain along the arrows $M_{u} M_{1}, T_{u}$ and $T_{1}$ in (b). The blue and magenta lines are the fitting plots of the strain distribution in the twin lamellae and the matrix, respectively.

\section{Note10. Dislocations blocked by TBs in the Au nanorode}




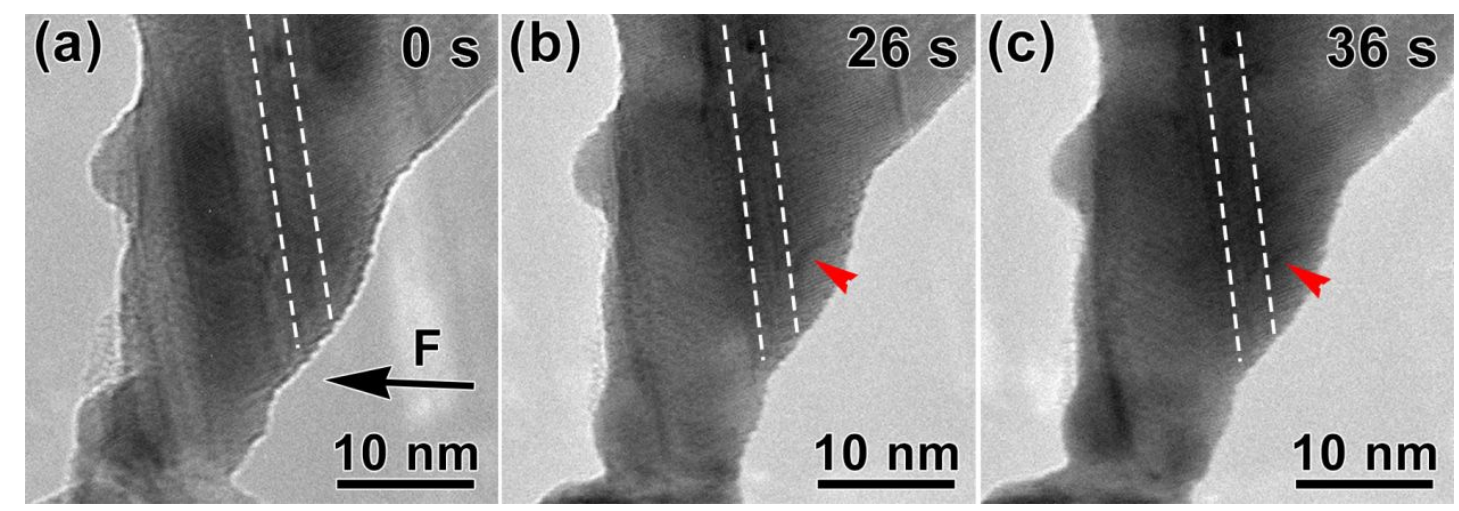

Figure S16. (a-c) In situ bright-filed TEM images show that a dislocation was blocked by the TB.

White dashed lines indicate TBs. The red arrows indicate the dislocation. The black arrow indicates the force direction.

\section{Supplementary Reference}

(1) Song, M.; Du, K.; Huang, Z.; Huang, H.; Nie, Z.; Ye, H. Deformation-Induced Dissolution and Growth of Precipitates in an Al-Mg-Er Alloy During High-Cycle Fatigue. Acta Materialia 2014, 81, 409-419.

(2) Song, M.; He, S.; Du, K.; Huang, Z.; Yao, T.; Hao, Y.; Li, S.; Yang, R.; Ye, H. Transformation Induced Crack Deflection in a Metastable Titanium Alloy and Implications on Transformation Toughening. Acta Materialia 2016, 118, 120-128.

(3) Rupert, T.; Gianola, D.; Gan, Y.; Hemker, K. Experimental Observations of StressDriven Grain Boundary Migration. Science 2009, 326 (5960), 1686-1690.

(4) You, Z.; Li, X.; Gui, L.; Lu, Q.; Zhu, T.; Gao, H.; Lu, L. Plastic Anisotropy and Associated Deformation Mechanisms in Nanotwinned Metals. Acta Materialia 2013, $61(1), 217-227$. 